\title{
Resultados do Registro de Cirurgias Colorretais Videolaparoscópicas Realizadas no Estado de Minas Gerais - Brasil de 1996 a 2009
}

\author{
Colorectal Laparoscopic Surgeries Performed in the State of Minas Gerais - \\ Brasil From 1996 to 2009
}

\begin{abstract}
FÁBIO LOPES DE QUEIROZ ${ }^{1}$, MARCELO GIUSTIWERNECK CÔRTES ${ }^{2}$, PAULO ROCHANETO $^{2}$, ADRIANACHEREM ALVES ${ }^{2}$, ANTÔNIO HILÁRIO ALVES FREITAS ${ }^{3}$, ANTONIO LACERDAFILHO ${ }^{1}$, AUGUSTO MOTTANEIVA ${ }^{2}$, BERNARDO HANAN $^{2}$, BRUNO GIUSTIWERNECK CÔRTES ${ }^{5}$, CHRISTIANE DE SOUZABECHARA ${ }^{2}$, CLEBER LUIZ SCHEIDEGGER MAIA JUNIOR ${ }^{2}$, CRISTIANE KOIZIMI MARTOS FERNANDES ${ }^{6}$, ELIANE SANDER MANSUR ${ }^{1}$, GERALDO MAGELA GOMES DACRUZ7 ${ }^{7}$, HÉLIO ANTÔNIO SILVA ${ }^{8}$, ISABELAALVARENGAMENDONÇA ${ }^{3}$,JANDER BAIRRAL VASCONCELOS ${ }^{9}$, JULIANO ALVES FIGUEIREDO ${ }^{8}$, KANTHYAARREGUY DE SENA, LEONARDO MACIEL, LUCIANA PYRAMO COSTA ${ }^{4}$, MAGDA MARIA PROFETADA LUZ $^{10}$, MARCO ANTÔNIO MIRANDADOS SANTOS ${ }^{2}$, MARIA ZULEIME CARMONA ${ }^{1}$, RAMON PIRES MARANHÃO $^{5}$, RODRIGO DE ALMEIDAPAIVA $^{1}$, RODRIGO GOMES DA SILVA $^{10}$, SINARA MÔNICADE OLIVEIRALEITE ${ }^{4}$, TEONAUGUSTO DE NORONHAOLIVEIRA ${ }^{2}$, THAÍSABARBOSADA SILVA $^{11}$, VALDIVINO ALVES FILHO $^{1}$, PAULOCESAR DE CARVALHOLAMOUNIER $^{1}$

${ }^{1}$ Coloproctologista do Hospital Felício Rocho, ${ }^{2}$ Residente de coloproctologia, ${ }^{3}$ Coloproctologista do Hospital da Policia Militar de Minas Gerais, ${ }^{4}$ Coloproctologista do Hospital Governador Israel Pinheiro, ${ }^{5}$ Coloproctologista do Hospital Life Center, ${ }^{6}$ Coloproctologista do Hospital Socor, ${ }^{7}$ Coloproctologista da Santa Casa de Belo Horizonte, ${ }^{8}$ Coloproctologista do Hospital da Baleia, ${ }^{9}$ Coloproctologista da Santa Casa de Juiz de Fora, ${ }^{10}$ Coloproctologista do Hospital das Clínicas - UFMG, ${ }^{11}$ Coloproctologista do Hospital Otaviano Neves.
\end{abstract}

QUEIROZ FL; CÔRTES MGW; ROCHA NETO P; ALVES AC; FREITAS AHA; LACERDA FILHO A; NEIVA AM; HANAN B; CÔRTES BGW; BECHARA CS; MAIA JUNIOR CLS; FERNANDES CKM; MANSUR ES; CRUZ GMG; SILVA HA; MENDONÇAIA; VASCONCELOS JB; FIGUEIREDO JA; SENA KA; MACIEL L; COSTA LP; LUZ MMP; SANTOS MAM; CARMONA MZ; MARANHÃO RP; PAIVA RA; SILVA RG; LEITE SMO; OLIVEIRA TAN; SILVA TB; ALVES FILHO V; LAMOUNIER PCC. Resultados do Registro de Cirurgias Colorretais Videolaparoscópicas Realizadas no Estado de Minas Gerais - Brasil de 1996 a 2009. Rev bras Coloproct, 2010;30(1): 061-067.

RESUMO: Introdução: A partir de 1991, a videolaparoscopia começou a ser considerada no tratamento de doenças colorretais. $O$ aprimoramento da técnica cirúrgica associado aos benefícios encontrados em diversos estudos publicados levou a modificações nas perspectivas da videolaparoscopia. A partir da publicação do estudo COST as ressecções oncológicas laparoscópicas foram reconhecidas como alternativa viável, com resultados semelhantes à cirurgia convencional. Pacientes e métodos: Realizou-se pesquisa através de formulário específico e consulta a prontuários dos principais serviços de coloproctologia de Belo Horizonte. Avaliando-se sexo, idade, indicação cirúrgica, procedimento realizado, técnica laparoscópica, complicações, conversão, estadiamento e recidiva (no caso de neoplasias). Resultados: Foram levantados dados sobre 503 cirurgias colorretais laparoscópicas: 347 (68,9\%) em mulheres e 156 (31,1\%) homens. A técnica cirúrgica foi totalmente laparoscópica em 137 casos, vídeo-assistida 245 casos. O procedimento mais realizado foi a retossigmoidectomia (41,1\%), seguido pela colectomia direita (12,5\%), colectomia esquerda $(6,9 \%)$. Doenças benignas foram responsáveis por $259(51,5 \%)$ casos, destes as principais indicações cirúrgicas foram endometriose 126 (48,6\%), pólipos 40 (15,4\%), doença diverticular 30 (11,6\%). Das 240 cirurgias realizadas por doenças malignas as mais frequentes foram retossigmoidectomia $102(42,5 \%)$, colectomia direita $46(19,1 \%)$, colectomia esquerda $18(7,5 \%)$, amputação abdominoperineal 18 (7,5\%). Houve 54 conversões $(10.7 \%)$ dos casos, $12,9 \%(31 / 240)$ nos casos de neoplasias, 8,5\% (22/259) nos de doenças benignas. Complicações sistêmicas ou cirúrgicas ocorreram em 31 (6,1\%) e 56 (11,1\%) casos, respectivamente. Foram registrados onze $(\mathbf{2 , 1 8 \%})$ óbitos nos primeiros 30 dias após a cirurgia. Conclusão: $O$ estudo atual foi o primeiro levantamento da implantação de cirurgias colorretais laparoscópicas realizado de forma multicêntrica em Minas Gerais. Os dados levantados são consistentes com registros nacionais de videocirurgia colorretal, mostrando a eficiência do método de aprendizado com realização de cirurgias com tutor. Além disso, que pequena parte das cirurgias colorretais são realizadas por via laparoscópica no estado, restritos apenas a centros especializados, sobrecarregando esses serviços e limitando o acesso para a população.

Descritores: Cirurgia colorretal; Videolaparoscopia; Videocirurgia.

Trabalho realizado no Hospital Felício Rocho - MG - Brasil.

Recebido em 09/10/2009

Aceito para publicação em 20/11/2009 


\section{INTRODUÇÃO}

A partir de 1991, iniciou-se o uso da videolaparoscopia no tratamento de doenças colorretais. $\mathrm{O}$ aprimoramento da técnica cirúrgica associado aos benefícios encontrados em diversos estudos publicados levou a modificações nas perspectivas da videolaparoscopia, sendo hoje reconhecida como técnica cirúrgica de escolha no tratamento de afecções benignas como pólipos e doença diverticular não complicada $^{2}$. Com o aumento da experiência, foi possível estender as possibilidades da laparoscopia. Passou a ser aceita a realização de cirurgia laparoscópica em pacientes obesos, naqueles com cirurgias abdominais prévias, em pacientes idosos e até em casos de urgência. Na doença de Crohn a laparoscopia tem se mostrado segura, além de apresentar melhor custo-benefício que a cirurgia aberta ${ }^{12}$.

Vários trabalhos foram realizados com o objetivo de avaliar a laparoscopia no tratamento das doenças malignas colorretais ${ }^{3,4,5,6,7,8,17}$. O câncer colorretal representa um problema importante de saúde pública. Estima-se que em 2008, a incidência dessa neoplasia no Brasil seja de 26.990 casos, sendo em Minas Gerais 2.160 casos. Desses, entre 480 a 679 ocorrendo na capital, com uma taxa de incidência de 16,41 casos por 100.000 habitantes do sexo masculino e 22,24 casos por 100.00 habitantes do sexo feminino ${ }^{1,13}$.

As ressecções laparoscópicas para câncer colorretal foram recentemente reconhecidas como alternativa viável, associada a resultados oncológicos semelhantes aos da colectomia convencional. Além de apresentar vantagens como menor dor e íleo paralítico pós-operatórios, menor taxa de infecção de ferida operatória, menor tempo de hospitalização, menor trauma operatório, menos imunossupressão pósoperatória, melhor resultado estético, melhor qualidade de vida no curto prazo, menor taxa de hérnias incisionais, e menor taxa de readmissão hospita$1 a r^{3,4,6,7,8,16,17,18,20,24,28}$. Há evidências da eficácia e segurança na ressecção do câncer de reto por laparoscopia, embora resultados em longo prazo ainda necessitem investigação $0^{5,9,10}$.

$\mathrm{O}$ aprendizado da videolaparoscopia exige um grande esforço do cirurgião, além de investimentos com a formação e adequação tecnológica. A curva de aprendizado é longa, sendo muitas vezes necessária a utilização de simuladores e cirurgias em animais, além de experiência em cirurgias por doenças benignas, antes da realização cirurgias para tratamento de tumores ${ }^{18,20,24,25}$.

Para o sucesso do procedimento, é necessária uma equipe integrada de profissionais treinados em cirurgia laparoscópica. A escolha da técnica anestésica adequada à cirurgia é fundamental para o bom desenvolvimento operatório. Além disso, durante a laparoscopia perde-se a sensação tátil, sendo importante realizar a localização adequada de tumores, muitas vezes recomendando-se a tatuagem colonoscópica prévia próximo à lesão. É considerado rotina o preparo de intestinal pré-operatório, visando facilitar a manipulação do cólon e intestino delgado durante o procedimento.

A escolha da técnica cirúrgica aberta ou laparoscópica deve ser individualizada e discutida com o paciente. É necessária uma avaliação global do paciente, levando em consideração suas características e comorbidades, a doença de base e a experiência do cirurgião com cada técnica ${ }^{20}$.

Apesar dos avanços da laparoscopia no Brasil, as cidades brasileiras possuem situações muito diferentes. Enquanto algumas regiões realizam um número grande de cirurgias, outras não as realizam. Esse trabalho tem por finalidade relatar a experiência dos diversos serviços de coloproctologia de Belo Horizonte e Minas Gerais em laparoscopia colorretal, comparando com levantamentos de dados de outras cidades brasileiras e com a literatura internacional. Fazendo, dessa forma, um diagnóstico da situação da cirurgia colorretal videolaparoscópica no estado.

\section{PACIENTES E MÉTODOS}

No início de 2009, foram enviados comunicados a todos os cirurgiões coloproctologistas inscritos na Sociedade Mineira de Coloproctologia (SMCP) por meio de e-mail e de pedido formal nas reuniões da SMCP para que reunissem os dados referentes aos pacientes submetidos à cirurgia colorretal videolaparoscópica em seus serviços. Foram coletados dados através de formulário específico. Posteriormente essas informações foram agrupadas em um único banco de dados e analisadas.

Foram recebidos dados de nove serviços. Os principais pontos avaliados foram: sexo, idade, indicação cirúrgica, procedimento realizado, técnica laparoscópica, complicações, conversão, estadiamento e recidiva (no caso de neoplasias). 


\section{RESULTADOS}

Foram levantados dados sobre 503 cirurgias colorretais laparoscópicas. Deste total 347 (68.9\%) eram de pacientes do sexo feminino e $156(31.1 \%)$ masculino. Retirados os casos de endometriose, 215 (58.0\%) dos casos foram de mulheres e $156(42.0 \%)$ de homens. A idade dos pacientes variou entre 13 e 86 anos, com média de 51,6 anos.

A técnica cirúrgica utilizada foi totalmente laparoscópicas em 137 (27.2\%) casos, vídeo assistida em $245(48.7 \%)$ casos, hand-assisted em um $(0.2 \%)$ e sem resposta 120 (23.9\%) casos. Em $274(54,4 \%)$ pacientes foi relatado preparo de colon pré-operatório.

O procedimento mais realizado por videolaparoscopia foi a retossigmoidectomia $(41,1 \%)$, seguido pela colectomia direita $(12,5 \%)$, colectomia esquerda $(6,9 \%)$ e colectomia parcial $(5,8 \%)$ - tabela 1 .

Houve 54 conversões para via laparotômica correspondendo a $10.7 \%$ dos casos. A taxa de conversão para os casos operados por neoplasias foi de 12,9\% (31/240), nas doenças benignas 8,5\% (22/259).

Em $31(6,1 \%)$ casos foram observadas complicações sistêmicas. Complicações cirúrgicas ocorreram em $56(11,1 \%)$ pacientes. Sendo as principais: 17 casos de fístulas anastomóticas $(3,3 \%), 12$ de sangramento $(2,4 \%)$ e nove de infecção de sítio cirúrgico $(1,8 \%)$. Onze $(2,18 \%)$ óbitos nos primeiros 30 dias após a cirurgia foram registrados, havendo variação de $1,5 \%$ em serviço com grande casuística a 14,3\% em serviço com experiência inicial. Os números de casos realizados por equipe de Coloproctologia foram variaram de 9 a 131 casos.

Conseguiu-se obter informações sobre a data de realização de 345 dos 503 procedimentos, destes dois $(0,6 \%)$ foram realizados em 2003 , oito $(2,3 \%)$ em

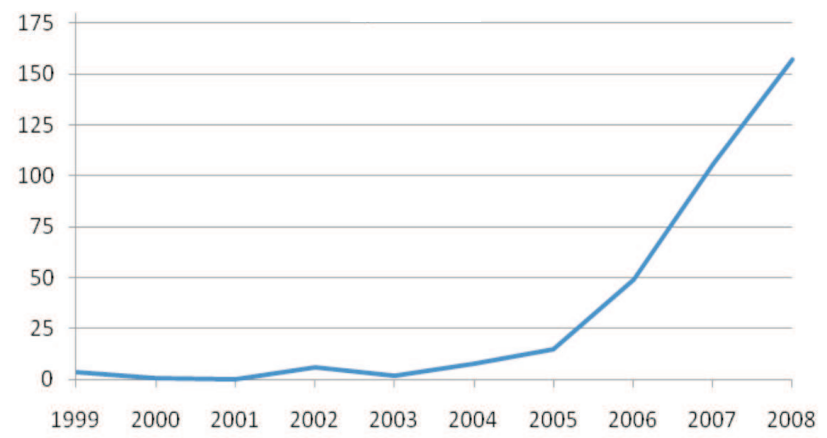

Gráfico 1 - Número de cirurgias laparoscópicas por ano.
2004, $15(4,3 \%)$ em 2005, $49(14,2 \%)$ em 2006, 104 $(30,1 \%)$ em 2007 e 148 (42,9\%) em 2008 - gráfico 1.

Doenças benignas foram responsáveis por 259 $(51,5 \%)$ casos, desses as principais indicações cirúrgicas foram endometriose $126(48,6 \%)$, pólipos 40 $(15,4 \%)$, doença diverticular $30(11,6 \%)$ e megacólon chagásico $17(6,6 \%)$ - tabela 2.

Os procedimentos realizados em pacientes com doenças benignas mais comuns foram sigmoidectomia ou retossigmoidectomia $118(45,9 \%)$,

Tabela 1 - Total de procedimentos realizados.

\begin{tabular}{lcr}
\hline Procedimentos & Número & \% \\
\hline Retossigmoidectomia & 207 & 41,1 \\
Colectomia direita & 63 & 12,5 \\
Colectomia esquerda & 35 & 6,9 \\
Excisão total do mesorreto & 31 & 6,2 \\
Colectomia parcial & 29 & 5,8 \\
Ressecção em disco & 27 & 5,4 \\
Colectomia total & 20 & 4,0 \\
Shaving & 19 & 3,8 \\
Amputação abdominoperineal & 19 & 3,8 \\
Sigmoidectomia & 19 & 3,8 \\
Proctocolectomia total & 12 & 2,4 \\
Operação de Duhamel & 10 & 2,0 \\
Outros & 12 & 2,4 \\
\hline
\end{tabular}

Tabela 2 - Indicações cirúrgicas parar doenças benignas.

\begin{tabular}{lcc}
\hline Indicação & Número & \% \\
\hline Endometriose & 126 & $48,6 \%$ \\
Pólipos & 40 & $15,4 \%$ \\
Doença diverticular & 30 & $11,6 \%$ \\
Megacólon chagásico & 17 & $6,6 \%$ \\
Polipose Adenomatosa Familiar & 12 & $4,6 \%$ \\
Tumores benignos & 8 & $3,1 \%$ \\
Prolapso retal & 5 & $1,9 \%$ \\
Lesões de crescimento lateral & 4 & $1,5 \%$ \\
Fístulas & 3 & $1,2 \%$ \\
Retocolite ulcerativa & 3 & $1,2 \%$ \\
Inércia colônica & 2 & $0,7 \%$ \\
Outros & 9 & $3,5 \%$ \\
& 259 & $100 \%$ \\
\hline
\end{tabular}


ressecção em disco $27(10,4 \%)$, shaving $19(7,3) \%$, colectomia direita $18(6,6 \%)$, colectomia esquerda 17 $(6,6 \%)$, colectomia parcial $12(4,6 \%)$ e colectomia total $12(4,6 \%)$ - tabela 3.

Os adenocarcinomas foram responsáveis por $233(46,3 \%)$ dos casos, sendo a localização mais frequente das lesões o cólon sigmóide e retossigmóide 104 (44,6\%), cólon direito 59 (26,1\%), reto $47(20,2 \%)$ e cólon transverso $6(2,5 \%)$ - tabela 4 .

Outros tumores malignos foram responsáveis por sete casos (quatro carcinomas de células escamosas de canal anal e três carcinóides).

Das 240 cirurgias realizadas por doenças malignas as mais frequentes foram retossigmoidectomia $102(42,5 \%)$, colectomia direita $46(19,1 \%)$, colectomia esquerda 18 (7,5\%), amputação abdominoperineal 18 $(7,5 \%)$, colectomia parcial $17(7,1 \%)$, excisão total do mesorreto $16(6,6 \%)$, colectomia total $8(3,3 \%)$, sigmoidectomia $5(2,1 \%)$, outras $10(4,2 \%)$ - tabela 5 .

\section{DISCUSSÃO}

Desde a primeira cirurgia colorretal videolaparoscópica realizada no estado em 1996, foram levantados dados sobre 503 casos.

A predominância de pacientes do sexo feminino, mesmo quando excluídos os casos de endometriose, no estudo está de acordo com o levantamento nacional realizado em $2007^{14}$.

A incidência total estimada de neoplasias colorretais foi 679 casos em Belo Horizonte para o ano de $2008^{13}$. Estima-se que desses, 93 casos foram operados por via laparoscópica. Considerando-se que em média até $95 \%(70-98 \%)^{16,18,19,22,23}$ das neoplasias colorretais são ressecáveis cirurgicamente de forma eletiva, é possível calcular-se um valor aproximado de $14,4 \%$ das abordagens das neoplasias colorretais sendo realizada por via laparoscópica. Esse valor é semelhante aos relatados em estudos populacionais internacionais entre $2,4 \%$ a $19,5 \%{ }^{16,20,21}$. Deve-se, entretanto, salientar que $15-20 \%$ das neoplasias se manifestam com quadro abdominal agudo, sendo considerada contra-indicada a ressecção laparoscópica ${ }^{18,19}$.

Quando se avalia a proporção de $4,5 \%$ de casos operados por laparoscopia em todo o estado, porém, percebe-se a grande centralização de cirurgias na capital. Esses dados evidenciam a dificuldade de acesso a essa tecnologia pela população em geral, levando os pacientes interessados muitas vezes a recor-
Tabela 3 - Procedimentos realizados em pacientes com doenças benignas.

\begin{tabular}{lcc}
\hline Procedimento & Número & \% \\
\hline Retossigmoidectomia & 207 & 41,1 \\
Sigmoidectomia ou & & \\
retossigmoidectomia & 119 & $45,9 \%$ \\
Ressecção em disco & 27 & $10,4 \%$ \\
Shaving & 19 & $7,3 \%$ \\
Colectomia direita & 17 & $6,6 \%$ \\
Colectomia esquerda & 17 & $6,6 \%$ \\
Colectomia parcial & 12 & $4,6 \%$ \\
Colectomia total & 12 & $4,6 \%$ \\
Duhamel & 10 & $3,9 \%$ \\
Outros & 26 & $10,0 \%$ \\
\cline { 2 - 3 } & 259 & $100 \%$ \\
\hline
\end{tabular}

Tabela 4 - Topografia dos adenocarcinomas.

\begin{tabular}{lrc}
\hline Topografia & Número & \% \\
\hline Sigmóide e retossigmóide & 104 & $44,6 \%$ \\
Cólon direito & 61 & $26,1 \%$ \\
Reto & 47 & $20,2 \%$ \\
Cólon transverso & 6 & $2,5 \%$ \\
Outros & 15 & $6,4 \%$ \\
Total & 233 & $100 \%$ \\
\hline
\end{tabular}

Tabela 5 - Cirurgias realizadas para neoplasias malignas.

\begin{tabular}{lcr}
\hline Cirurgia realizada & Número & \% \\
\hline Retossigmoidectomia & 102 & 42,5 \\
Colectomia direita & 46 & 19,1 \\
Colectomia esquerda & 18 & 7,5 \\
Amputação abdominoperineal & 18 & 7,5 \\
Colectomia parcial & 17 & 7,1 \\
Excisão total do mesorreto & 16 & 6,6 \\
Colectomia total & 8 & 3,3 \\
Sigmoidectomia & 5 & 2,1 \\
Outras & 10 & 4,2 \\
\hline & 240 & $100 \%$ \\
\hline
\end{tabular}


rer a centros especializados localizados em outras cidades, gerando uma sobrecarga nesses serviços e podendo acarretar atraso no tratamento de pacientes com neoplasia ${ }^{16,20}$.

A taxa de conversão encontrada foi de $10.7 \%$. Os dados da literatura mostram grande variação de 0 a $46 \%$ de acordo com idade, estado cardiopulmonar, presença de quadro inflamatório, obesidade, cirurgia prévia, localização e tamanho do tumor, estadiamento local da doença e experiência do cirurgião $0^{16,24,26,27,28}$. Com o aumento da experiência há tendência de estabilização da taxa abaixo de $10 \%{ }^{10}$.

Cirurgias videolaparoscópicas por neoplasias apresentaram uma tendência a maior frequência de conversões, $12,9 \% \times 8,5 \%$, não encontrando significância estatística $(\mathrm{p}=0,11)$. Uma explicação possível é a maior complexidade desses procedimentos, necessitando de margens oncológicas adequadas e por vezes ressecções de órgãos adjacentes em bloco. Assim como, distorção da anatomia e dificuldade de identificação de estruturas. Os dados encontrados na literatura, não evidenciam diferença na taxa de conversão ${ }^{21}$, e essa tendência pode se dever à amostra relativamente pequena.

As complicações tanto sistêmica quanto cirúrgica foram pouco frequentes na série avaliada, tendo ocorrido em 31 e 56 casos, respectivamente. Das complicações sistêmicas as mais freqüentes foram o íleo pós-operatório em dez casos, sepse ou choque séptico com oito casos.

O índice de morbidade pós-operatória foi de $17,3 \%$, que se mostra consistente com o levantamento nacional de $14,5 \%$ entre 2001 e 2007 . A principal complicação cirúrgica foi fístula/deiscência anastomótica com 17 (3,3\%) casos registrados. Lesões inadvertidas de vísceras são complicações temidas, especialmente quando não são percebidas durante o ato cirúrgico, na série relatada esses eventos ocorreram em $5(1 \%)$ casos.

A taxa de mortalidade total nos primeiros 30 dias após a cirurgia foi de $2,18 \%$, próximo à média nacional até 2001 de 1,6\%, mas acima da média para o período atual de $0,9 \%$. Devendo-se considerar a grande variação entre os serviços avaliados $(1,5 \%$ a $14,3 \%)$.

Com o aumento do número de procedimentos e da experiência dos cirurgiões colorretais, ocorreu aumento do número de procedimentos maiores e mais complexos. Isso se percebe através do nú- mero crescente de cirurgias como excisão total do mesorreto, amputações abdominoperineais. As neoplasias malignas foram responsáveis por $47,7 \%$ do total de cirurgias, refletindo a capacidade dos serviços na realização de cirurgias oncologicamente adequadas por via laparoscópica. Foram confirmados somente 2 casos de recidiva em 144 pacientes, com taxa de $1,38 \%$.

A videolaparoscopia colorretal começou a ser introduzida em sua maior parte pela demonstração de cirurgias por cirurgiões de outros estados e pela realização de cursos assistidos. Esse estudo comprova a eficiência desse método com o auxílio de tutores na introdução da cirurgia laparoscópica, consistente com relatos da literatura ${ }^{16,18,20}$.

\section{CONCLUSÃO}

No estudo atual foram levantados dados de instituições com casuísticas muito diferentes, algumas com experiência de mais de 10 anos outras ainda em fase inicial. $\mathrm{O}$ número de casos entre as instituições variou entre 9 e 131. A análise de todos os casos em banco de dados único não reflete a diferença entre os serviços, devendo a interpretação ser feita de forma cuidadosa.

Os dados do estudo foram adquiridos de forma retrospectiva pela revisão de prontuários e registros das equipes, devido à não uniformidade de informações e muitas vezes a registros pontuais, em muitos casos não foi possível avaliar pós-operatório tardio ou recidivas tumorais.

O estudo atual foi o primeiro levantamento de cirurgias colorretais laparoscópicas realizado de forma multicêntrica em Minas Gerais, encontrando as barreiras comuns nesse tipo de levantamento, como técnicas padronizadas de forma diferente, protocolos diferentes de acompanhamento e o registro ou não de dados.

Além de relatar a experiência em Belo Horizonte e Minas Gerais visamos com o estudo incentivar os serviços a manterem registros atualizados e completos das cirurgias laparoscópicas para facilitar a realização de pesquisas e acompanhamentos de resultados cirúrgicos.

A técnica laparoscópica de abordagem colorretal é uma tecnologia recente, dispendiosa e que apresenta curva de aprendizado longa, esses fatores limitaram seu desenvolvimento no es- 
tado de Minas Gerais concentrando-se na capital e em poucos centros com os recursos necessários. Uma pequena proporção das cirurgias colorretais atualmente são realizadas por essa via quando se analisa todo o estado, o que leva a sobrecarga de serviços que a utilizam e a uma de- manda reprimida de pacientes que não têm acesso a essa tecnologia. É do interesse dos autores a divulgação dessa modalidade cirúrgica de forma a motivar a criação de serviços descentralizados capazes de realizar a videolaparoscopia colorretal em todo o estado.

ABSTRACT: Introduction: Since 1991, laparoscopic surgery started to be considered in the treatment of colorectal disease. With the improvement of the technique and the confirmation of the benefits shown in several studies, the perspective of laparoscopy has changed. Recently, the oncologic resections were recognized as a viable alternative, with similar results to the conventional technique. Patients and methods: Data were collected based on charter review and a specific form from the coloproctology reference centers in Minas Gerais. The data assessed were age, gender, indication for surgery, the procedure performed, the laparoscopic technique, complications, conversion rate and staging (in case of tumor). Results: Data from 503 surgeries were analyzed: 347 (68.9\%) were women, and $156(31.1 \%)$ men. The technique was totally laparoscopic in 137 cases, laparoscopicallyassisted in 245 . The procedure most frequently performed was rectosigmoidectomy $(\mathbf{4 1 . 1 \%})$, followed by right hemicolectomy $(12.5 \%)$ and left hemicolectomy (6.9\%). Conversion occurred in $10.7 \%$ (54) cases, being $12.9 \%(31 / 240)$ in cases of neoplasia and 8.5\% (22/259) in benign diseases. Systemic or surgical complications occurred in $31(6.1 \%)$ and $56(11.1 \%)$ cases, respectively. The surgical mortality was $2.18 \%$ (11). Benign diseases were responsible for $259(51.5 \%)$ cases, the most frequent indications were endometriosis $126(48.6 \%)$, polyps $40(15.4 \%)$ and diverticular disease $30(11.6 \%)$ cases. From the 240 surgeries performed for malignant disease, the most frequent were rectosigmoidectomy $102(42.5 \%)$, right hemicolectomy $46(19.1 \%)$, left hemicolectomy 18 (7.5\%) and abdominoperineal resection 18 (7.5\%). Conclusion: This study was the first survey about the implementation of laparoscopic colorectal surgery done in Minas Gerais. The data are consistent with the national registry of colorectal surgery.

Key words: Colorectal surgery; Videolaparoscopy; Videosurgery.

\section{REFERÊNCIAS}

1 - INCA. Instituto Nacional do Câncer. Ministério da Saúde: câncer no Brasil, estimativas 2008 e 2009. http:// www.inca.gov.br/estimativa/2008 (accessed June 26, 2009).

2 - Rafferty J, Shellito P, Hyman NH, Buie WD. Standards Committee of American Society of Colon and Rectal Surgeons. Practice parameters for sigmoid diverticulitis. Dis Colon Rectum, 2006;49(7):939-44.

3 - Lacy AM, Garcia-Valdecasas LC, Delgado S et al. Laparoscopy-assisted colectomy versus open colectomy for treatment of non-metastatic colon cancer: a randomized trial. Lancet, 2002;359:2224-2229.

4 - Lacy A. Colon cancer: laparoscopic resection. Ann Oncol, 2005;16:88-92.

5 - Guillou PJ, Quirke P, Thorpe H et al. Short-term endopoints of conventional versus laparoscopic-assisted surgery in patients with colorectal cancer (MRC CLASICC trial): multicentre, randomized controlled trial. Lancet, 2005;365:1718-1726.

6 - The Colon Cancer Laparoscopic or Open Resection Study Group (COLOR). Laparoscopic surgery versus open surgery for colon cancer: short-term outcomes of a randomized trial. Lancet Oncol, 2005;6:477-484
7 - Nelson H, Sargent DJ, Wieand HS, Fleshman J et al. The Clinical Outcomes of Surgical Therapy Study Group. A comparison of laparoscopically assisted and open colectomy for colon cancer. N Engl J Med, 2004;350:2050-2059.

8 - Bonjer HJ, Hop WC, Nelson H et al. Laparoscopically assisted VS open colectomy for colon cancer: a meta-analysis. Arch Surg, 2007; 142(3): 298-303.

9 - Delgado S, Momblan D, Salvador L et al. Laparoscopic-assisted approach in rectal cancer patients: lessons learned from $>200$ patients. Surg Endosc, 2004;18:1457-1462.

10 - Morino M, Parini U, Giraudo G et al. Laparoscopic total mesorectal excision: a consecutive series of 100 patients. Ann Surg, 2003;237:335-342.

11 - Stulberg JJ, Champagne BJ, Horan M et al. Emergency laparoscopic colectomy: does it measure up to open? The American Journal of Surgery, 2009;197,296-301.

12 - Misika S, Iannelli A et al. Can laparoscopy reduce hospital stay in the treatment of crohn's diseases? Dis Colon Rectum, 2001;44:1661-1666.

13 - DATASUS - Ministério da Saúde: www.datasus.gov.br

14 - Valarini R, Campos FGCM. Resultados do registro nacional brasileiro em vídeo-cirurgia colorretal - 2007. Rev Bras Coloproctol, 2008;28(2):145-155.

15 - Junior HJ. Untreated colorectal cancer in a community hospital. J Surg Oncology, 2006;29(3):198-200. 
16 - Romano G, Gagliardi G et al. Laparoscopic colorectal surgery: why it is still not the gold standard and why it should be. Tech Coloproctol, 2008;12:185-188.

17 - Fleshman J, Sargent DJ, Green E. Laparoscopic Colectomy for Cancer Is Not Inferior to Open Surgery Based on 5-Year Data From the COST Study Group Trial Annals of Surgery. 2007;246(4):655-664.

18 - National Institute for Clinical Excellence. Laparoscopic Surgery for Colorectal Cancer Appraisal No 17. Submission by The Association of Coloproctology of Great Britain and Ireland July 2005.

19 - Pavlidis TE, Marakis G, Ballas K et al. Does Emergency Surgery Affect Resectability of Colorectal Cancer? Acta chir belg, 2008;108:219-225.

20 - The National Training Programme in Laparoscopic Colorectal Surgery http://www.lapco.nhs.uk/about-the-programme.php

21 - Harinath G, Shah PR, Haray PN, Foster ME. Laparoscopic colorectal surgery in Great Britain and Ireland - Where are we now? Colorectal Dis, 2005;7(1):86-89.

22 - Leon-Rodríguez E, Hernández MC. Colon cancer at the National Institute of Nutrition. I. Treatment results from 1979 to 1989 . Rev Invest Clin, 1996;48(3):191-198.

23 - Cruz GMG, Santana JL, Santana SKAA et al. Câncer Colônico - Epidemiologia, Diagnóstico, Estadiamento e Gradação
Tumoral de 490 Pacientes. Rev bras Coloproct, 2007;27(2):139-153

24 - Dowson HM, Huang A, Soon Y et al. Systematic Review of the Costs of Laparoscopic Colorectal Surgery. Dis Colon Rectum, 2007;50(6):908-919.

25 - Schlachta CM, Mamazza J, Seshadri PA et al. Defining a Learning Curve for Laparoscopic Colorectal Resections. Dis Colon Rectum, 2001;44(2):217-222.

26 - Tekkis PP, Senagore AJ, Delaney CP. Conversion rates in laparoscopic colorectal surgery. A predictive model with 1253 patients. Surg Endosc, 2005;19:47-54.

27 - Belizon A, Sardinha CT, Sher ME. Converted laparoscopic colectomy. What are the consequences? Surg Endosc, 2006;20:947-951.

28 - Veldkamp R, Gholghesaei M, Bonjer HJ et al. Laparoscopic resection of colon cancer Consensus of the European Association of Endoscopic Surgery (E.A.E.S.). Surg Endosc, 2004;18:1163-1185.

Endereço para correspondência: FÁBIO LOPES DE QUEIROZ

Av. Contorno, 9530 - Barro Preto

Belo Horizonte - MG

30110-130 - Coloproctologia

E-mail: fabiolopesq@hotmail.com 